\title{
Association of Serum Uric Acid with Family History of Coronary Artery Disease in a Pakistani Population
}

\author{
Sabir Hussain $^{1}$, Tahir Iqbal ${ }^{2}$ \\ ${ }^{1}$ Department of Biosciences, COMSATS Institute of Information Technology, Islamabad, Pakistan \\ ${ }^{2}$ Department of Internal Medicine, Shifa College of Medicine, Shifa International Hospital, Islamabad, Pakistan
}

\section{To the Editor,}

Coronary artery disease (CAD) or coronary atherosclerosis is a major cause of death and disability around the world. Exploring the complex nature of CAD is not an easy and simple task. In fact, CAD is a heritable trait and has remained the focus of advanced research for the last few decades. Multiple factors including hyperlipidemia, hypertension, diabetes mellitus, family history, and genetics are all assumed to be involved in the pathogenesis of CAD (1). Understanding all of the established and novel emerging risk factors by clinicians is critical in order to prevent cardiovascular disabilities. Despite the clear involvement of established risk factors, clinical studies have shown that serum uric acid (SUA) is associated with hypertension or other cardiovascular diseases (2). The pathological role of elevated SUA in CAD either independently or in association with other cardiovascular disease risk factors has been the topic of debate. Recently, a Mendelian-randomized study showed a positive relationship between body mass index, hyperuricemia and elevated SUA (3). However, the increased serum uric acid and its association with a family history of CAD has not been published. Therefore, the aim of the present study was to investigate the association of SUA with a family history of CAD.

The present study protocol is adherent to the Helsinki Declaration of 1975 as revised in 1997 and was approved by the Ethical Committee, COMSATS Institute of Information Technology, Islamabad. A total of 330 subjects consisting of 110 patients with CAD (group 1), 110 unrelated healthy subjects without a family history of CAD (group 2), and 110 healthy controls with a family history of CAD in at least three generations (group 3) were analysed. Biochemi- cal analyses of lipid profile, and SUA were determined by using the AMP Diagnostics kits (Austria) with the help of a Vitalab Selectra E chemistry analyzer (Netherlands). The inclusion criterion was applied for both the patients and controls in this study. Study subjects were evaluated by physical and clinical history, ECG, and coronary angiography. The results were evaluated by using Chi-square and One-Way ANOVA.

The basic and clinical variables are listed in Table 1. Lipid biomarkers were significantly associated with CAD in the study population. SUA levels were $5.7 \pm 1.20$ in CAD patients, $5.5 \pm 1.0$ in controls with CAD family history and $3.8 \pm 0.65$ in healthy subjects with no family history. SUA was significantly increased in CAD patients compared with controls with no family history $(5.7 \pm 1.20 \mathrm{mg} / \mathrm{dL}$ vs $3.8 \pm 0.65 \mathrm{mg} / \mathrm{dL}$, $\mathrm{p}<0.0001)$. The comparison of SUA was not significantly different between $\mathrm{CAD}$ patients and controls with a positive family history of CAD $(5.7 \pm 1.20$ vs $5.5 \pm 1.0, \mathrm{p}=0.235)$. Beside, a significant difference was observed between the subjects with a family history of CAD and those with no such history (5.5 \pm 1.0 vs $3.8 \pm 0.65, \mathrm{p}<0.0001)$.

The pathological role of elevated SUA in CAD has been suggested by several possible mechanisms (2). The underlying atherosclerotic mechanism may involve abnormal xanthine oxidase, oxidative stress, and the formation of free radicals causing a deleterious effect on endothelial cell function. Increased SUA stimulates inflammation and promotes platelet adhesiveness (4). However, recent data regarding the association of SUA with CAD in a dependent or independent manner is not very clear (5). In our study, for the first time, we have established a significant association of SUA with a family history of CAD, and this should be considered a risk factor for $\mathrm{CAD}$ in this high risk population. 
TABLE 1. Baseline and clinical characteristics of study population $(n=330)$

\begin{tabular}{lccccc}
\hline Parameters & Group 1 & Group 2 & Group 3 & F-value( *p Value) & F-value(**p Value) \\
\hline Age (years) & $49.5 \pm 16.3$ & $47.3 \pm 13.9$ & $45.4 \pm 15.9$ & $1.160(0.283)^{\mathrm{a}}$ & $0.890(0.346)^{\mathrm{a}}$ \\
BMI $\left(\mathrm{kg} / \mathrm{m}^{2}\right)$ & $26.5 \pm 3.1$ & $26.3 \pm 3.1$ & $25.7 \pm 2.8$ & $0.229(0.633)^{\mathrm{a}}$ & $2.269(0.133)^{\mathrm{a}}$ \\
Male/Female (n) & $77 / 33$ & $77 / 33$ & $77 / 33$ & $1.00^{\mathrm{b}}$ & $1.00^{\mathrm{b}}$ \\
SBP (mmHg) & $147.4 \pm 18.6$ & $118.3 \pm 7.8$ & $118.8 \pm 9.2$ & $228.9(<0.0001)^{\mathrm{a}}$ & $0.189(0.664)^{\mathrm{a}}$ \\
DSBP(mmHg) & $94.9 \pm 9.8$ & $77.6 \pm 7.5$ & $79.3 \pm 7.8$ & $216.2(<0.0001)^{\mathrm{a}}$ & $2.71(0.101)^{\mathrm{a}}$ \\
Cholesterol (mg/dL) & $198.2 \pm 42.2$ & $146.3 \pm 35.8$ & $181.5 \pm 39.9$ & $96.7(<0.0001)^{\mathrm{a}}$ & $47.4(<0.0001)^{\mathrm{a}}$ \\
Triglyceride (mg/dL) & $122.4 \pm 36.3$ & $102.0 \pm 36.3$ & $117.4 \pm 52.9$ & $17.4(<0.0001)^{\mathrm{a}}$ & $6.338(0.013)^{\mathrm{a}}$ \\
LDL(mg/dL) & $94.5 \pm 23.1$ & $82.9 \pm 18.8$ & $85.7 \pm 22.4$ & $16.6(<0.0001)^{\mathrm{a}}$ & $1.008(0.316)^{\mathrm{a}}$ \\
HDL (mg/dL) & $35.7 \pm 8.9$ & $39.7 \pm 9.2$ & $36.1 \pm 8.1$ & $10.7(0.001)^{\mathrm{a}}$ & $9.488(0.002)^{\mathrm{a}}$ \\
Uric acid (mg/dL) & $5.7 \pm 1.20$ & $3.8 \pm 0.65$ & $5.5 \pm 1.0$ & $213.8(<0.0001)^{\mathrm{a}}$ & $223.5(<0.0001)^{\mathrm{a}}$ \\
\hline Vas
\end{tabular}

Values are given as $\pm \mathrm{SD}$ and numbers

Group $1=$ CAD patients $(\mathrm{n}=110)$, Group 2 = Controls without CAD family history $(\mathrm{n}=110)$, Group 3 = Controls with CAD family history ( $\mathrm{n}=110)$, *P Value for Group 1 vs Group 2 , **P Value for Group 2 vs Group 3, SBP systolic blood pressure, DSBP diastolic blood pressure ap Value calculated by using the One-Way ANOVA

bP Value calculated by Pearson Chi-square test

Ethics Committee Approval: Ethics committee approval was received for this study from the ethics committee of COMSATS Institute of Information Technology, Islamabad.

Informed Consent: Written informed consent was obtained from patients who participated in this study.

Peer-review: Externally peer-reviewed.

Author contributions: Concept - S.H., T.I.; Design - S.H.; Supervision - S.H.; Resource - S.H., T.I.; Materials - S.H.; Data Collection \&/or Processing - T.I.; Analysis \&/or Interpretation - S.H., T.I.; Literature Search - S.H.; Writing - S.H. Critical Reviews - S.H., T.I.

Acknowledgments: We are thankful to all the study participants. Special thanks for Majeed Ali in helping the biochemical analysis.

Conflict of Interest: No conflict of interest was declared by the authors.

Financial Disclosure: The authors declared that this study has received no financial support.

\section{REFERENCES}

1. Youhanna S, Platt DE, Rebeiz A, Lauridsen M, Deeb ME, Nasrallah A,et al; FGNETCARD consortium. Parental consanguinity and family history of coronary artery disease strongly predict early stenosis. Athersclerosis 2010;212:559-63. [CrossRef]

2. Kang DH. Potential role of uric Acid as a risk factor for cardiovascular disease. Korean J Intern Med 2010;25:18-20. [CrossRef]

3. Palmer TM, Nordestgaard BG, Benn M, Tybjaerg-Hansen A, Davey Smith G, Lawlor DA, Timpson NJ. Association of plasma uric acid with ischaemic heart disease and blood pressure: mendelian randomisation analysis of two large cohorts. BMJ 2013;347:f4262 [CrossRef]

4. Kanellis J, Watanabe S, Li JH, Kang DH, Li P, Nakagawa T, et al. Uric acid stimulates monocyte chemo-attractant protein-1 in vascular smooth muscle cells via mitogen- activated protein kinase and cyclooxygenase-2. Hypertension 2003;41:1287-93. [CrossRef]

5. Skak-Nielsen H, Torp-Pedersen C, Finer N, Caterson ID, Van Gaal L, James WP, Maggioni AP, Sharma AM, Coutinho W, Andersson C. Uric Acid as a Risk Factor for Cardiovascular Disease and Mortality in Overweight/Obese Individuals. PLoS One 2013;8:e59121. [CrossRef] 